\title{
Review Article \\ Dyslipidemia Might Be Associated with an Increased Risk of Osteoarthritis
}

\author{
Jianping Xiong, ${ }^{1}$ Junyu Long, ${ }^{1} \mathrm{Xi}$ Chen, ${ }^{1}$ Ye Li $\mathbb{D},{ }^{1,2}$ and Hai Song $\mathbb{D}^{3}$ \\ ${ }^{1}$ Department of Surgery, Peking Union Medical College Hospital, Chinese Academy of Medical Sciences and Peking Union Medical \\ College (CAMS \& PUMC), Beijing 100730, China \\ ${ }^{2}$ Department of Orthopedics, Peking Union Medical College Hospital, Chinese Academy of Medical Sciences and Peking Union \\ Medical College (CAMS \& PUMC), Beijing 100730, China \\ ${ }^{3}$ Department of Science and Education, Tangshan People's Hospital, Tangshan 063000, China
}

Correspondence should be addressed to Ye Li; liye@pumch.cn and Hai Song; songhz2018@163.com

Received 23 September 2019; Revised 9 December 2019; Accepted 7 January 2020; Published 17 February 2020

Guest Editor: Chaohong Li

Copyright (C) 2020 Jianping Xiong et al. This is an open access article distributed under the Creative Commons Attribution License, which permits unrestricted use, distribution, and reproduction in any medium, provided the original work is properly cited.

\begin{abstract}
Background. According to several studies, the autoimmune response may lead to osteoarthritis and dyslipidemia and may affect the homeostasis of the human body's internal environment and then cause its own immune regulation. Consequently, the risk of osteoarthritis might be increased by dyslipidemia, but this association is not universally acknowledged. Therefore, a systematic review and meta-analysis was conducted to study the relationship between dyslipidemia and the risk of osteoarthritis. Methods. In this study, PubMed, EMBASE, and the ISI Web of Science were used to identify related studies published before July 2018. The relationship between dyslipidemia and the risk of osteoarthritis was evaluated on the basis of relative risk (RR) values and the corresponding 95\% confidence intervals (CIs). To further investigate this relationship, we also employed the random effects model proposed by DerSimonian and Laird. Results. A total of nine studies were included to study the effect of dyslipidemia on the risk of osteoarthritis, including four cohort, three case-control, and two cross-sectional studies. Among these studies, six stated data for knee osteoarthritis, two reported on hand osteoarthritis, and one reported on hip osteoarthritis. A total of 53,955 participants were included in the meta-analysis, comprising 22,501 patients with OA (19,733 hand OA, 2,679 knee OA, and 89 hip OA). Based on the meta-analysis of case-control and cross-sectional studies, osteoarthritis was clearly higher in those with dyslipidemia compared to those who did not suffer from dyslipidemia (case-control: $\mathrm{OR}=1.37 ; 95 \% \mathrm{CI}=1.27-1.46$; crosssectional: $\mathrm{OR}=1.33 ; 95 \% \mathrm{CI}=1.21-1.46)$. In addition, the meta-analysis of cohort studies did not present any relationship between dyslipidemia and $\mathrm{OA}(\mathrm{RR}=1.00 ; 95 \% \mathrm{CI}=0.85-1.14)$. Conclusions. Even though our meta-analysis of case-control and cross-sectional studies suggested a strong relationship between dyslipidemia and osteoarthritis; this relationship was not validated by our meta-analysis of only cohort studies. As a result, further investigation needs to be conducted on the relationship between dyslipidemia and osteoarthritis, considering the significant public health relevance of the topic.
\end{abstract}

\section{Introduction}

Osteoarthritis $(\mathrm{OA})$ refers to a chronic degenerative disease that involves the cartilage, as well as its surrounding tissues [1]. OA is considered the most common joint disease, and nearly $10-12 \%$ of the population suffers from OA [2]. In addition, it is expected that this number will increase dramatically due to the quickly increasing aging population combined with the growing prevalence of obesity [3]. Consequently, osteoarthritis is considered to have a negative influ- ence on the health economy [4]. It can be forecast that by the year 2032, an additional 26,000 per million patients over the age of 45 will present to their general practitioner with osteoarthritis compared to 2012 [5]. OA is associated with age, female gender, obesity, joint injury, and career, as well as a high level of physical activity [5]. In addition, the autoimmune response of the synovium plays an important role in rheumatoid arthritis. In recent years, the immunological pathogenesis of synovium in osteoarthritis has attracted the attention of many researchers. Whether the immune 
mechanism and inflammatory mediators are involved in the occurrence and development of osteoarthritis deserves further discussion. This may provide a new research idea for the pathogenesis of osteoarthritis, to improve our understanding of the development of this disease and change the way of treatment. In recent years, research has shown that metabolic syndrome is closely associated with OA, which is even a part of generalized metabolic disorder. Metabolic syndrome is composed of a bundle of interrelated metabolic risk factors, including diabetes, obesity, dyslipoproteinemia, and hypertension [6]. Furthermore, the incidence of metabolic syndrome is very high; it has been estimated to be as high as $26.7 \%$ in industrialized countries [6]. In the context of musculoskeletal disorders, metabolic syndrome has increasingly gained more attention because of its relationship with knee OA [7]. Obesity, the main feature in metabolic syndrome, is overwhelmingly related to degenerative joint changes in regard to mechanical load [8]. Alternatively, obesity-related $\mathrm{OA}$ can afflict nonweight-bearing joints (e.g., the hands), signifying a role of adipokines (circulating mediators released by adipose tissue), such as leptin. Thus, OA may have a systemic metabolic element [9]. In addition, OA can be categorized into three phenotypes: metabolic OA, age-related OA, and injury-related OA [10]. Nevertheless, as one of the components of metabolic syndrome, the role of dyslipidemia in the pathogenesis of $\mathrm{OA}$ is not completely understood. Dyslipidemia may affect the homeostasis of the human body's internal environment and then cause its own immune regulation. Dyslipidemia is related to chronic lowgrade inflammation and oxidative stress, likely increasing the development of OA $[11,12]$. A survey carried out by Ghandehari concluded that approximately 51.4 million US adults presented with high cholesterol and triglycerides, in addition to 36.1 million with elevated low-density lipoproteins [13]. As a result, we chose to conduct a systematic review and meta-analysis of the published observational studies to better comprehend the relationship between dyslipidemia and the risk of OA.

\section{Materials and Methods}

This research was performed according to the Preferred Reporting Items for Systematic Reviews and Meta-Analyses (PRISMA) Statement [14] and the Meta-analysis of Observational Studies in Epidemiology (MOOSE) guidelines [15].

2.1. Data Sources and Search Strategy. Published studies in PubMed, EMBASE, and the Web of Science were searched based on the following keywords: "hyperlipidaemia" OR "dyslipidemia" OR "triglyceride" OR "cholesterol" OR "lipoprotein" OR "lipid" OR "metabolic syndrome") and ("OA"). No restrictions on language or the date of publication were placed. Additionally, this study also searched the reference lists. Unpublished studies and original data were not included.

2.2. Eligibility Criteria for Study Selection. The eligibility criteria were as follows: study design (randomized controlled trials and cohort, case-control, or cross-sectional studies); an exposure factor of blood lipid levels and an outcome of
OA; availability of the odds ratio $(\mathrm{OR}) /$ risk ratio $(\mathrm{RR})$ values and corresponding 95\% confidence intervals (CIs) for dyslipidemia patients and the general population; or the availability of sufficient information to measure these variables. The most recent all-inclusive study was searched under the condition that two studies used the same population. The definition of dyslipidemia was in line with the US National Cholesterol Education Program Adult Treatment Panel III guidelines. In accordance with the National Cholesterol Education Program, the definition of dyslipidemia was highdensity lipoprotein cholesterol (HDL-C) $<40 \mathrm{mg} / \mathrm{dL}$, as well as total cholesterol, low-density lipoprotein cholesterol (LDL-C), and TG levels of $\geq 200, \geq 130$, and $\geq 130 \mathrm{mg} / \mathrm{dL}$, respectively [5]. The definition of osteoarthritis was in line with the American College of Rheumatology (ACR) clinical and clinical plus radiographic criteria [6]. The ACR classification criteria for (OA) permits the categorization of individuals for hand, knee, and hip OA [6]. We strictly abided by this classification standard.

2.3. Data Abstraction and Quality Assessment. Two scholars (J.X. and J.L.) obtained the essential information from the chosen studies according to the standard. The following information was gathered: name of the first author, publication year, country in which the research was carried out, study design, number of participants, period of follow-up, sources of controls, potential adjusted confounding variables, OR/RR values, and 95\% CIs.

To date, no available common scale has been proposed to evaluate the quality of all kinds of observational studies. As a result, two authors individually employ the modified Newcastle-Ottawa Scale (NOS) [16] as reported by Zhu et al. [17] to assess the quality of the included studies. Quality types were allocated in accordance with the scores of each study, consisting of high quality (score 7-9), medium quality (score 4-6), and low quality (score less than 4) [18]. The maximum total score could reach 9 points, and discrepancies were solved by mutual agreement.

2.4. Statistical Analysis. The random effects model put forward by DerSimonian and Laird was applied to investigate the relationship between dyslipidemia and the risk of OA among the cohort studies [19]. The $I^{2}$ statistic was employed to evaluate heterogeneity between the studies. Low, medium, and high heterogeneities were categorized as 25\%,50\%, and $75 \%$, respectively [20]. Definite heterogeneity was assumed if the $p$ value was less than 0.1 . Sensitivity analyses were conducted by altering the pooling model [21]. In addition, a sensitivity analysis was carried out to evaluate the influence of each individual study on the summarized estimate by means of successively excluding one research study at a time. Publication bias was assessed using Begg's [22] and Egger's [23] tests. No testing for funnel plot asymmetry was carried out due to the limited number of studies included in the analysis $(n<10)$ [24].

Furthermore, we conducted a meta-analysis of the casecontrol and cross-sectional studies in regard to the influence of dyslipidemia on the risk of OA and expressed the results as 


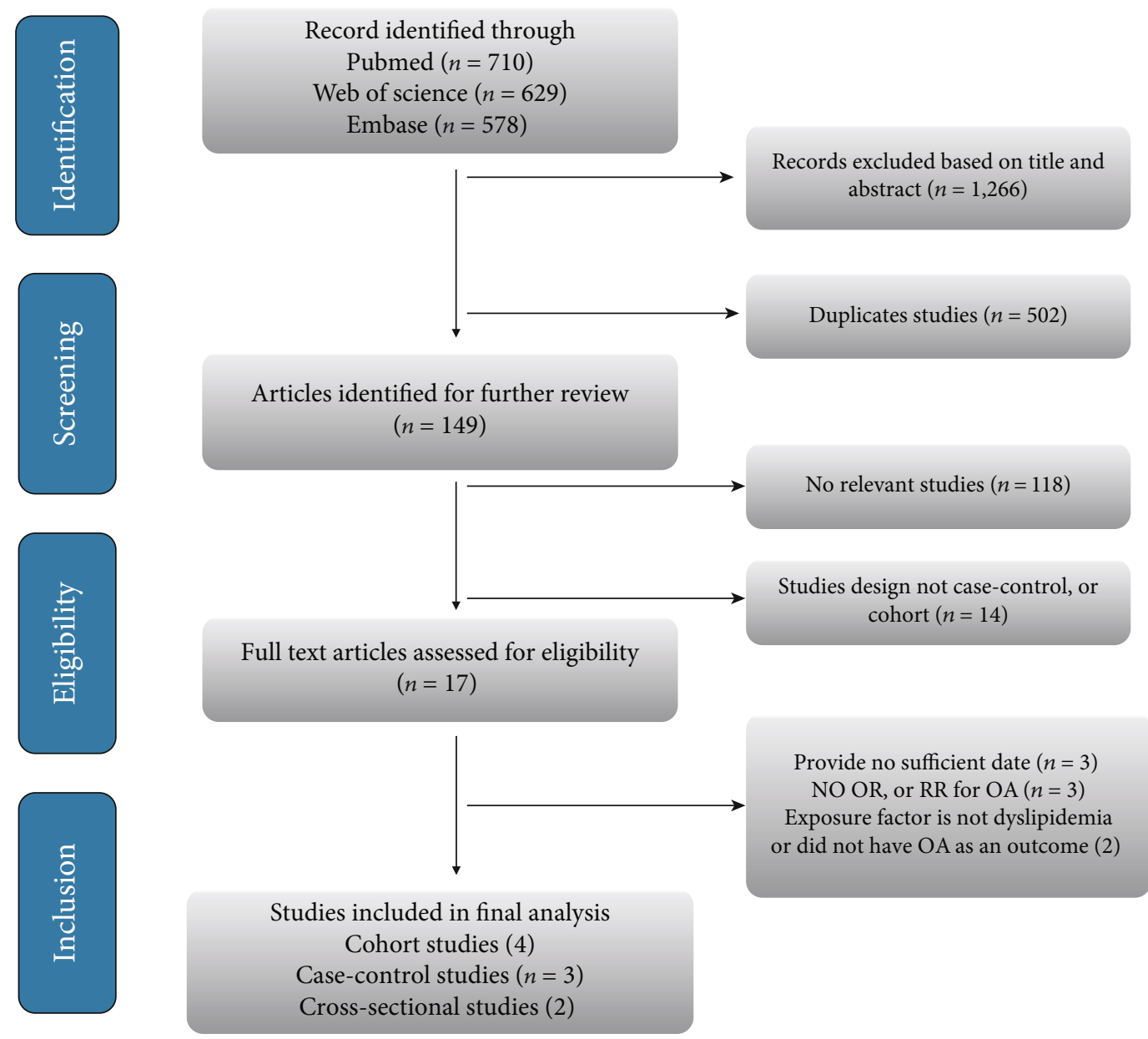

FIGURE 1: Study selection process for the meta-analysis.

pooled risk ratios with $95 \%$ CIs with the application of a random effects model.

STATA version 12.0 (Stata) was carried out to perform all statistical analyses.

\section{Results}

3.1. Study Selection and Study Characteristics. The process of study selection for the meta-analysis can be found in Figure 1. In total, 1,917 articles were obtained through the initial search, and 502 were duplicates. An additional 1,266 studies were removed based on the title and abstract. Eventually, after evaluation of the full texts, eight studies were excluded for the reason that they did not satisfy our inclusion criteria: three studies offered inadequate information [25-27], three studies did not offer ORs or RRs for $\mathrm{OA}$ or adequate information to calculate these variables [28-30], and two studies were removed that either did not have dyslipidemia as an exposure or did not have OA as an outcome [31, 32]. Ultimately, nine available observational articles were recognized for our meta-analysis [33-41].

Table 1 presents the principal features of the studies included in the meta-analysis, all of which were observational studies. The studies were conducted in the following countries: two in China, one in the UK, one in Germany, one in
Switzerland, one in Sweden, one in Australia, one in Japan, and one in Korea. Four studies were cohort studies, three were case-control studies, and two were cross-sectional studies. All the studies included the levels of serum TC and TGs, as well as LDL and HDL cholesterol as exposures. A total of 53,955 participants were included in the metaanalysis, comprising 22,754 patients with OA (19,733 hand $\mathrm{OA}, 2,679$ knee OA, and 89 hip OA). The mean age of patients ranged from 46.5 to 81.2 years, and the presence of hypertension and diabetes mellitus was described by $17.5 \%$ to $79.3 \%$ and $7.1 \%$ to $48.5 \%$ of patients, respectively. Data from 1988 to 2014 were collected. The follow-up period was within the range of 1 to 13 years. The modified NOS scores for the included studies ranged from 5 to 9 , including seven high-quality studies and two mediumquality studies (Table 2). A large proportion of the studies offered risk estimates that were adjusted for age (6 studies), smoking (6 studies), gender (4 studies), physical activity (4 studies), and alcohol consumption (3 studies). Fewer studies were adjusted for lipid-lowering agents/statins (2 studies) and BMI (1 study) (Table 1).

3.2. Cohort Studies. Reports from four studies permitted the calculation of effect estimates for OA [34-36, 38]. All of the cohort studies were population based, and the follow-up 


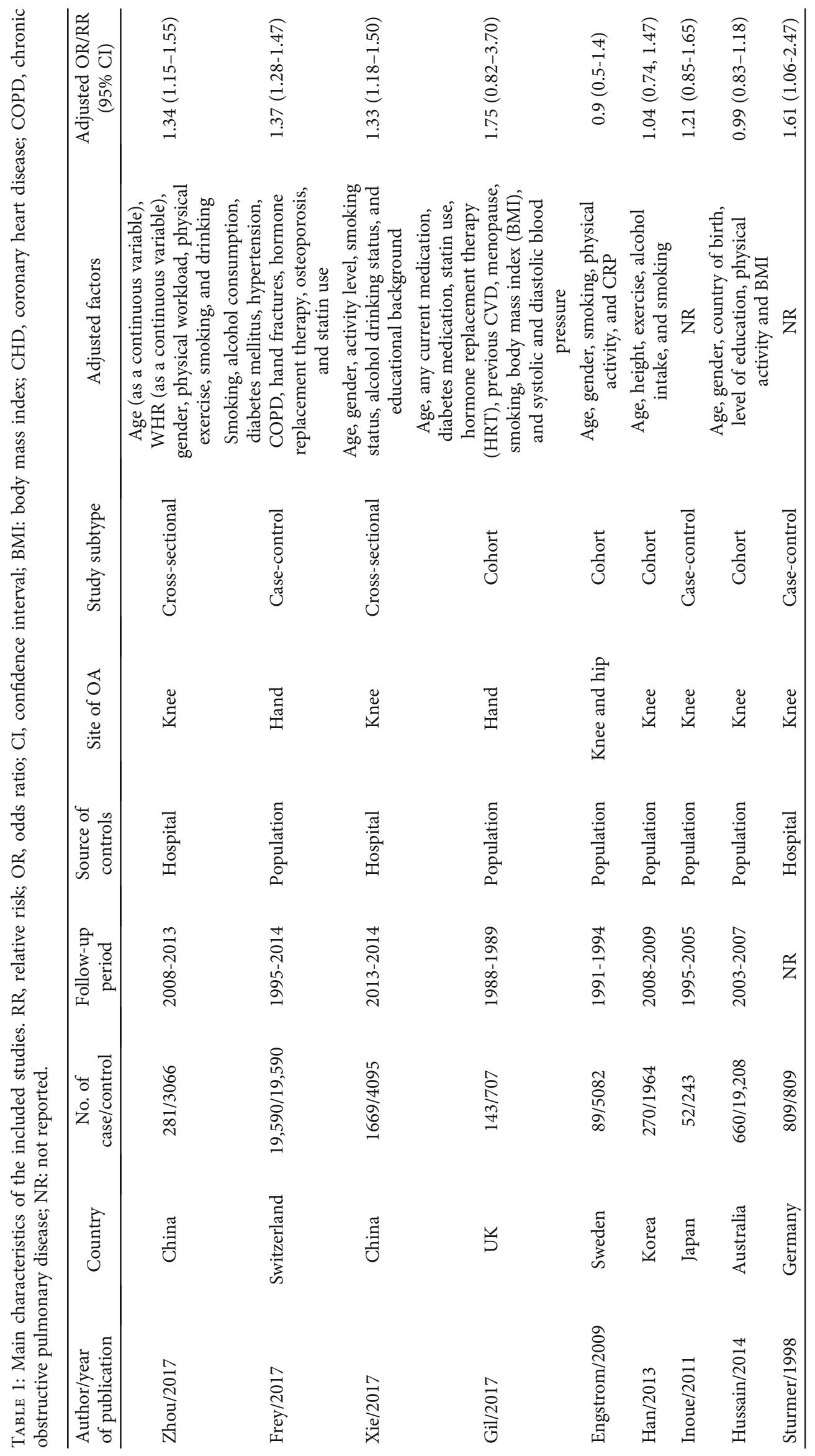


TABle 2: Modified Newcastle-Ottawa Scale scores for the included studies. The asterisks represent a score (number of stars).

\begin{tabular}{|c|c|c|c|c|c|c|c|c|c|}
\hline $\begin{array}{l}\text { Author/year } \\
\text { of publication }\end{array}$ & $\begin{array}{l}\text { Fully } \\
\text { defined } \\
\text { cases }\end{array}$ & $\begin{array}{l}\text { Defines } \\
\text { the study } \\
\text { design }\end{array}$ & $\begin{array}{l}\text { Selection } \\
\text { of controls }\end{array}$ & $\begin{array}{c}\text { Describes } \\
\text { the general } \\
\text { characteristics }\end{array}$ & $\begin{array}{l}\text { Controlled for } \\
\text { important } \\
\text { factors or } \\
\text { confounding } \\
\text { factors }\end{array}$ & $\begin{array}{l}\text { Lists of inclusion } \\
\text { and exclusion } \\
\text { criteria for all } \\
\text { participants }\end{array}$ & $\begin{array}{l}\text { Provides } \\
\text { enrollment } \\
\text { duration } \\
\text { for all } \\
\text { participants }\end{array}$ & $\begin{array}{l}\text { Indicates } \\
\text { study period } \\
\text { and follow- } \\
\text { up duration }\end{array}$ & $\begin{array}{l}\text { Total } \\
\text { score }\end{array}$ \\
\hline Zhou/2017 & * & * & $*$ & * & $* *$ & * & $*$ & * & 9 \\
\hline Frey/2017 & * & $*$ & & * & $* *$ & $*$ & $*$ & * & 8 \\
\hline Xie/2017 & * & * & * & * & $* *$ & * & * & & 8 \\
\hline Gil/2017 & * & & * & * & $* *$ & $*$ & & * & 7 \\
\hline Engstrom/2009 & * & * & & * & * & * & * & * & 7 \\
\hline $\mathrm{Han} / 2013$ & * & $*$ & $*$ & * & * & $*$ & $*$ & * & 8 \\
\hline Inoue/2011 & * & * & & * & & & * & $*$ & 5 \\
\hline Hussain/2014 & $*$ & $*$ & $*$ & & $*$ & $*$ & * & $*$ & 7 \\
\hline Sturmer/1998 & $*$ & $*$ & * & & & $*$ & $*$ & & 5 \\
\hline
\end{tabular}

was in the range of 1 to 3 years. Among them, two studies reported data for knee $\mathrm{OA}$, one study reported on hand $\mathrm{OA}$, and one study reported on hip and knee OA. In the pooled analysis, dyslipidemia exerted a null influence on the risk of $\mathrm{OA}\left(\mathrm{RR}=1.00 ; 95 \% \mathrm{CI}=0.85-1.14 ; I^{2}=0 \%\right)$ (Figure 2). In the sensitivity analysis, the general results for the relationship between dyslipidemia and OA kept steady with the changes of the pooling model (fixed: $R R=1.00$; $95 \% \mathrm{CI}=0.85-1.14)$. In addition, when we successively removed each study in turn to evaluate the stability of the results, we found that no study likely influenced the pooled risk estimate (Figure 3). No test for funnel plot asymmetry was carried out due to the limited number of included studies $(n<10)$. Nevertheless, Begg's $(p=0.697)$ and Egger's $(p=0.465)$ tests failed to identify substantial publication bias.

3.3. Case-Control Studies. The relationship between dyslipidemia and the risk of OA was investigated by three casecontrol studies [33, 40, 41]. Two case-control studies were population-based, and one case-control study was hospital based. According to Frey et al. [33] and Sturmer et al. [41], there exists an increase in the risk of knee OA among dyslipidemia patients. Nevertheless, the results obtained by Inoue et al. [40] suggested that dyslipidemia does not obviously change the risk of hand OA. There exist significant pooled estimates of the effect of cirrhosis without any considerable heterogeneity $\quad\left(\mathrm{OR}=1.37 ; \quad 95 \% \mathrm{CI}=1.27-1.46 ; \quad I^{2}=0 \%\right)$ (Figure 2). Based on the general results, the risk of OA was obviously higher among dyslipidemia patients in comparison with the general population. In accordance with the sensitivity analysis, despite excluding studies where the sources of controls were not hospital-based, the relationship between dyslipidemia and OA remained steady. In addition, the general results regarding the relationship between dyslipidemia and OA were kept under the condition that the pooling model was changed (fixed effects model: OR = 1.37; $95 \% \mathrm{CI}=1.27-1.46$; random effects model: $\mathrm{OR}=1.37$; $95 \% \mathrm{CI}=1.27-1.46)$. Finally, when we successively removed each study in turn to evaluate the stability of the results, no research likely influenced the pooled risk estimate.
3.4. Cross-Sectional. Two hospital-based, cross-sectional studies reported the influence of dyslipidemia on the risk of OA [37, 39]. Both studies reported data on knee OA, and both reported an obvious connection of dyslipidemia with OA. The random effects meta-analysis also demonstrated that dyslipidemia considerably increased the risk of $\mathrm{OA}$, without heterogeneity $(\mathrm{OR}=1.33 ; 95 \% \mathrm{CI}=1.21-1.46$; $I^{2}=0 \%$ ) (Figure 2).

\section{Discussion}

Admittedly, this is the first meta-analysis to investigate the relationship between dyslipidemia and the risk of OA. Even though a meta-analysis of cohort studies did not present any connection between dyslipidemia and OA, a meta-analysis of case-control and cross-sectional studies demonstrated that the risk of OA was obviously higher among those suffering from dyslipidemia compared to those without dyslipidemia.

The potential pathophysiological mechanisms accounting for these results remain unknown. Changed expression of cholesterol influx genes in human osteoarthritic chondrocytes and in the cartilage of patients with OA has been depicted [42]. Moreover, in vivo studies have demonstrated that decreased high-density lipoprotein cholesterol plays an important role in the pathogenesis of OA. Based on early research, dietary cholesterol intake has been shown to increase spontaneous cartilage damage in mice [43]. Correspondingly, high LDL levels stimulate synovial inflammation, as well as ectopic bone formation, in mouse OA models [43]. LDL could be involved in the development and progression of $\mathrm{OA}$ through the stimulation of synovial cells and chondrocytes [44].

There are several strengths of our study. First, this is the first meta-analysis to investigate the relationship between dyslipidemia and the risk of $\mathrm{OA}$ with a large sample size (22,754 cases of OA and 53,955 participants), probably providing a reference in regard to this relationship. Clinically, the results indicate that patients with dyslipidemia are required to focus more on their risk of $\mathrm{OA}$ in comparison 


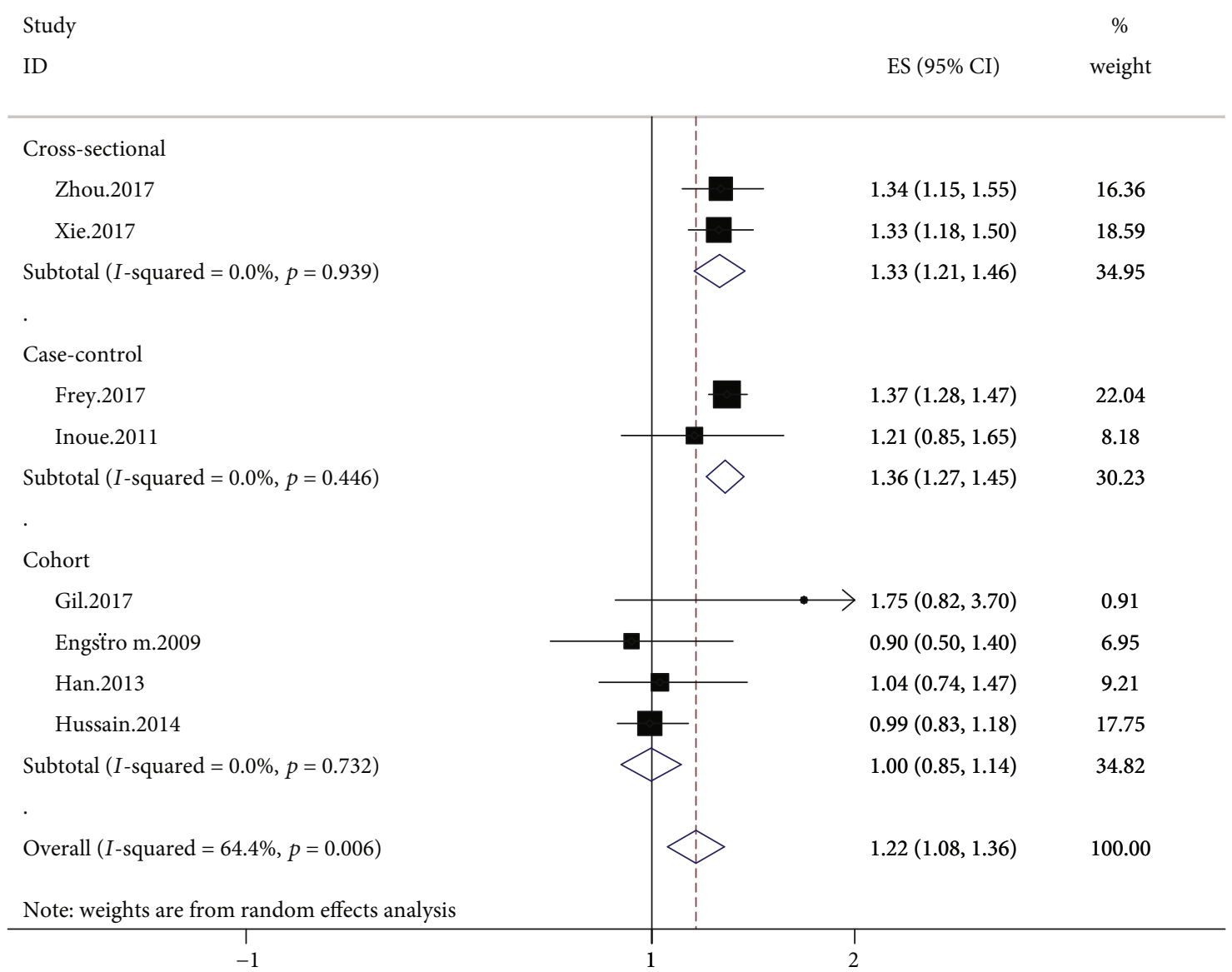

FIGURE 2: Forrest plot showing the relationship between dyslipidemia and the risk of OA, using a random effects model and depicted on a logarithmic scale. Squares represent the risk estimates for each individual study. Horizontal lines represent the 95\% confidence intervals, and diamonds represent the summary risk estimates with $95 \%$ confidence intervals. CI: confidence interval; ES: effect size.

with the general population. Second, a comprehensive literature search was conducted using PubMed, EMBASE, and the Web of Science to recognize potential studies from which to examine the relationship between dyslipidemia and the risk of OA. Additionally, most of the studies included were high-quality studies. All of these features enhance the reliability of our study.

However, this study still has some limitations. First, a meta-analysis of the different locations of OA, such as the knee, hip, and hand, was not conducted due to the limited number of included studies. The effect of dyslipidemia on the risk of knee vs. hip OA may have differences because of the diverse susceptibility of these two joints to metabolic factors. The knee may have more dependence on soft tissue and neuromuscular control for its stability. Comparatively, in the hip, the bony shape and joint congruence seem to have greater significance on the development of hip OA, which makes the hip less vulnerable to the effects of inflammation. Second, the observed outcome was an association, which is considered a confounding bias. Seven studies reported data on knee or hip osteoarthritis, but not all of the studies were adjusted for BMI or physical activity. Zhou et al. [39], Xie et al. [37], Engstrom et al. [35], Han et al. [36], and Monira Hussain et al. [34] reported on knee or hip osteoarthritis adjusted for physical activity (activity level). Hussain et al. [34] reported on knee osteoarthritis adjusted for BMI. Two studies (Inoue et al. [40] and Sturmer et al. [41]) that reported data on knee osteoarthritis did not adjust for BMI or physical activity. There were few data on knee OA patients compared with normal participants, because the studies by Inoue et al. [40] and Sturmer et al. [41] are aimed at investigating the data of the general population. Even though most studies were adjusted for recognized risk factors for OA, such as age, alcohol consumption, and smoking, many potential adjustment factors remained unclear, such as cardiovascular disease and obesity, showing a close association with the development of OA. Only two studies in the meta-analysis adjusted the OR/RR with lipid-lowering agents/statins. Additionally, we did not succeed in obtaining information concerning medication use, especially regarding lipid-lowering agents such as statins, which could have an influence on the development of OA. It has been indicated that statins might play a protective role in developing OA, likely caused by pleiotropic anti-inflammatory properties or by enhancing chondrogenesis $[45,46]$. Third, a meta-analysis of the effects of various kinds of dyslipidemia, such as low-density lipoprotein levels, triglyceride levels, and cholesterol levels, was not carried out due to the limited number of included studies. 


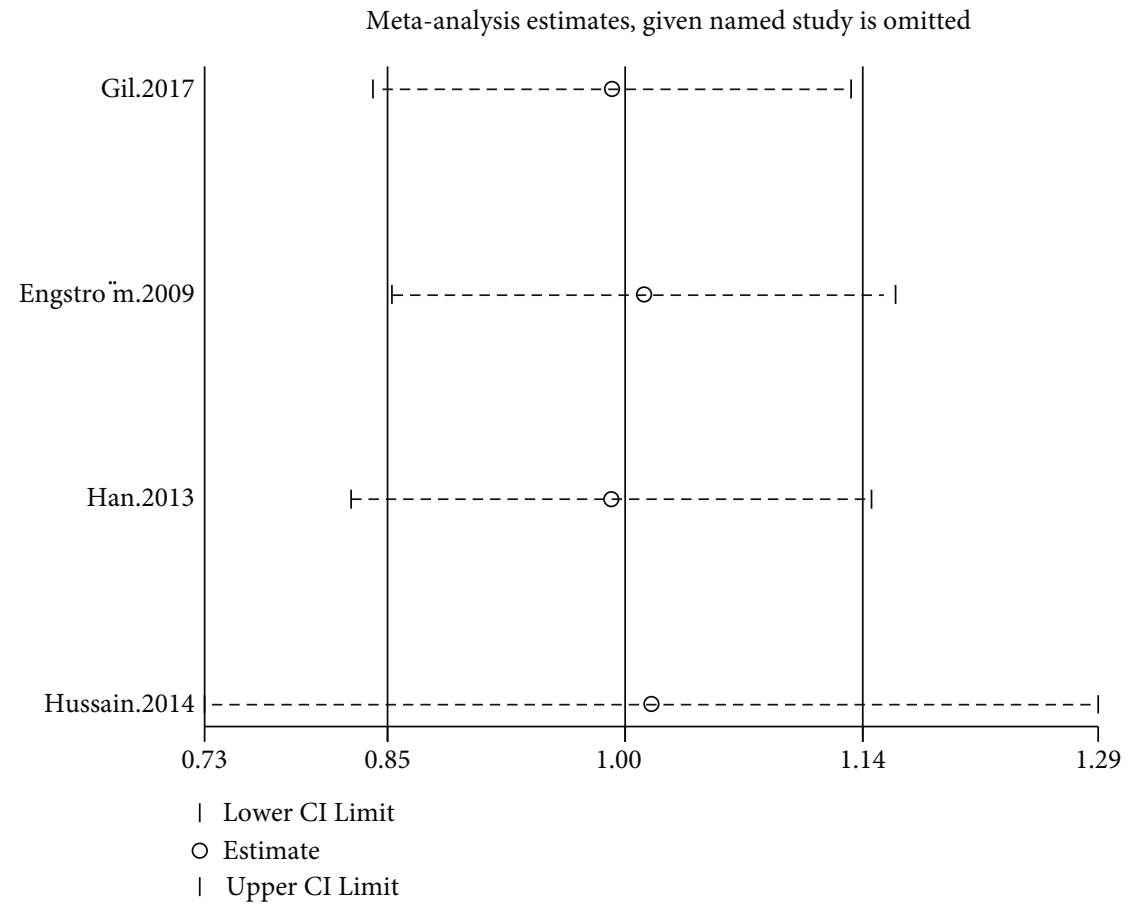

FIGURE 3: Sensitivity analysis of the association between dyslipidemia and the risk of OA (cohort study).

Ultimately, a large proportion of the included studies were cohort studies, yet we also included two cross-sectional studies and three case-control studies. Case-control studies tend to have recall and selection biases, and cross-sectional studies are insufficient for assessing the relationship between cirrhosis and the risk of stroke. It must also be admitted that a meta-analysis cannot deal with the limitations of the included studies. Finally, it is difficult to determine the causality of this relationship on the basis of these observational studies alone given the heterogeneity of the results and the limited data from prospective studies, which were restricted to 4 studies that, respectively, included $89,270,143$, and 660 cases of OA, as well as the lack of information about time-related factors. Although we were not always able to ascertain the source of the heterogeneity, we performed several sensitivity and subgroup analyses to address this issue.

In conclusion, considering there was a lack of evidence from the cohort studies but that strong connections were noted in the case-control and cross-sectional studies, the current systematic review and meta-analysis offers inadequate but fascinating evidence of the effects of dyslipidemia on the risk of OA. In general, the findings indicate that dyslipidemia might exert a significant pathogenic role in the development of OA, as well as offers a rationale for the shared care of patients by metabolic physicians. To be specific, structured clinical trials with predefined criteria for patient selection are still required to investigate the role of lipid-lowering therapies in the management of OA.

\section{Conflicts of Interest}

The authors declare that they have no conflicts of interest.

\section{Authors' Contributions}

J.X. designed the study and completed the manuscript. J.X. and J.L. searched the databases and reviewed the studies. X.C. and J.X. collected and analyzed the data. Y.L. and H.S. coordinated and provided financial the support for the proposed study. The final manuscript was read and approved by all the authors. Y.L. is the guarantor for the current study. Thanks are due to Simon C. Robson of Harvard Medical School Liver Center and Transplant Institute for helping us modify the language of the manuscript. Jianping Xiong, Junyu Long, and Xi Chen contributed equally to this work.

\section{References}

[1] P. S. Hsu, H. H. Lin, C. R. Li, and W. S. Chung, "Increased risk of stroke in patients with osteoarthritis: a population-based cohort study," Osteoarthritis and Cartilage, vol. 25, no. 7, pp. 1026-1031, 2017.

[2] P. Suri, D. C. Morgenroth, and D. J. Hunter, "Epidemiology of osteoarthritis and associated comorbidities," $P M \& R$, vol. 4, Supplement 5, pp. S10-S19, 2012.

[3] A. J. Hall, B. Stubbs, M. A. Mamas, P. K. Myint, and T. O. Smith, "Association between osteoarthritis and cardiovascular disease: systematic review and meta-analysis," European Journal of Preventive Cardiology, vol. 23, no. 9, pp. 938-946, 2016.

[4] R. Bitton, "The economic burden of osteoarthritis," The American Journal of Managed Care, vol. 15, Supplement 8, pp. S230-S235, 2009.

[5] R. C. Lawrence, D. T. Felson, C. G. Helmick et al., "Estimates of the prevalence of arthritis and other rheumatic conditions in the United States. Part II," Arthritis and Rheumatism, vol. 58, no. 1, pp. 26-35, 2008. 
[6] E. S. Ford, W. H. Giles, and A. H. Mokdad, "Increasing prevalence of the metabolic syndrome among U.S. adults," Diabetes Care, vol. 27, no. 10, pp. 2444-2449, 2004.

[7] R. Gandhi, F. Razak, P. Tso, J. R. Davey, and N. N. Mahomed, "Asian ethnicity and the prevalence of metabolic syndrome in the osteoarthritic total knee arthroplasty population," The Journal of Arthroplasty, vol. 25, no. 3, pp. 416-419, 2010.

[8] S. P. Messier, R. F. Loeser, G. D. Miller et al., "Exercise and dietary weight loss in overweight and obese older adults with knee osteoarthritis: the Arthritis, Diet, and Activity Promotion Trial," Arthritis and Rheumatism, vol. 50, no. 5, pp. 15011510, 2004.

[9] E. Yusuf, R. G. Nelissen, A. Ioan-Facsinay et al., “Association between weight or body mass index and hand osteoarthritis: a systematic review," Annals of the Rheumatic Diseases, vol. 69, no. 4, pp. 761-765, 2010.

[10] J. W. J. Bijlsma, F. Berenbaum, and F. P. J. G. Lafeber, "Osteoarthritis: an update with relevance for clinical practice," The Lancet, vol. 377, no. 9783, pp. 2115-2126, 2011.

[11] E. Esteve, W. Ricart, and J. M. Fernandez-Real, "Dyslipidemia and inflammation: an evolutionary conserved mechanism," Clinical Nutrition, vol. 24, no. 1, pp. 16-31, 2005.

[12] A. Kontush, E. C. de Faria, S. Chantepie, and M. J. Chapman, "A normotriglyceridemic, low HDL-cholesterol phenotype is characterised by elevated oxidative stress and HDL particles with attenuated antioxidative activity," Atherosclerosis, vol. 182, no. 2, pp. 277-285, 2005.

[13] H. Ghandehari, S. Kamal-Bahl, and N. D. Wong, "Prevalence and extent of dyslipidemia and recommended lipid levels in US adults with and without cardiovascular comorbidities: the National Health and Nutrition Examination Survey 20032004," American Heart Journal, vol. 156, no. 1, pp. 112-119, 2008.

[14] D. Moher, A. Liberati, J. Tetzlaff, and D. G. Altman, "Preferred reporting items for systematic reviews and meta-analyses: the PRISMA Statement," Open Medicine, vol. 3, no. 3, pp. e123e130, 2009.

[15] D. F. Stroup, J. A. Berlin, S. C. Morton et al., "Meta-analysis of observational studies in epidemiology: a proposal for reporting. Meta-analysis Of Observational Studies in Epidemiology (MOOSE) group," Journal of the American Medical Association, vol. 283, no. 15, pp. 2008-2012, 2000.

[16] A. Stang, "Critical evaluation of the Newcastle-Ottawa scale for the assessment of the quality of nonrandomized studies in meta-analyses," European Journal of Epidemiology, vol. 25, no. 9, pp. 603-605, 2010.

[17] W. Zhu, Y. F. Meng, Y. Wu, M. Xu, and J. Lu, “Association of alcohol intake with risk of diabetic retinopathy: a metaanalysis of observational studies," Scientific Reports, vol. 7, no. 1, p. 4, 2017.

[18] Y. P. Zhang, W. Q. Li, Y. L. Sun, R. T. Zhu, and W. J. Wang, "Systematic review with meta-analysis: coffee consumption and the risk of gallstone disease," Alimentary Pharmacology \& Therapeutics, vol. 42, no. 6, pp. 637-648, 2015.

[19] R. DerSimonian and N. Laird, "Meta-analysis in clinical trials," Controlled Clinical Trials, vol. 7, no. 3, pp. 177-188, 1986.

[20] J. P. T. Higgins and S. G. Thompson, "Quantifying heterogeneity in a meta-analysis," Statistics in Medicine, vol. 21, no. 11, pp. 1539-1558, 2002.

[21] J. Xiong, J. Lin, A. Wang et al., “Tea consumption and the risk of biliary tract cancer: a systematic review and dose-response meta-analysis of observational studies," Oncotarget, vol. 8, no. 24, pp. 39649-39657, 2017.

[22] C. B. Begg and M. Mazumdar, "Operating characteristics of a rank correlation test for publication bias," Biometrics, vol. 50, no. 4, pp. 1088-1101, 1994.

[23] M. Egger, G. Davey Smith, M. Schneider, and C. Minder, "Bias in meta-analysis detected by a simple, graphical test," $B M J$, vol. 315, no. 7109, pp. 629-634, 1997.

[24] J. A. C. Sterne, A. J. Sutton, J. P. A. Ioannidis et al., "Recommendations for examining and interpreting funnel plot asymmetry in meta-analyses of randomised controlled trials," $B M J$, vol. 343, no. 1, article d4002, 2011.

[25] D. Shin, "Association between metabolic syndrome, radiographic knee osteoarthritis, and intensity of knee pain: results of a national survey," The Journal of Clinical Endocrinology and Metabolism, vol. 99, no. 9, pp. 3177-3183, 2014.

[26] Q. Zhuo, W. Yang, J. Chen, and Y. Wang, "Metabolic syndrome meets osteoarthritis," Nature Reviews Rheumatology, vol. 8, no. 12, pp. 729-737, 2012.

[27] S. Le Clanche, D. Bonnefont-Rousselot, E. Sari-Ali, F. Rannou, and D. Borderie, "Inter-relations between osteoarthritis and metabolic syndrome: a common link?," Biochimie, vol. 121, pp. 238-252, 2016.

[28] A. E.-M. A. Afifi, R. M. Shaat, O. M. Gharbia, Y. E. Boghdadi, E. MME, and O. A. El-Emam, "Osteoarthritis of knee joint in metabolic syndrome,” Clinical Rheumatology, vol. 37, no. 10, pp. 2855-2861, 2018.

[29] K. Tootsi, A. Martson, J. Kals, K. Paapstel, and M. Zilmer, "Metabolic factors and oxidative stress in osteoarthritis: a case-control study," Scandinavian Journal of Clinical and Laboratory Investigation, vol. 77, no. 7, pp. 520-526, 2017.

[30] C. Erturk, M. A. Altay, A. Bilge, and H. Celik, "Is there a relationship between serum ox-LDL, oxidative stress, and PON1 in knee osteoarthritis?," Clinical Rheumatology, vol. 36, no. 12, pp. 2775-2780, 2017.

[31] R. Gandhi, K. M. Woo, M. G. Zywiel, and Y. R. Rampersaud, "Metabolic syndrome increases the prevalence of spine osteoarthritis," Orthopaedic Surgery, vol. 6, no. 1, pp. 23-27, 2014.

[32] A. Sharma, D. Gopalakrishnan, R. Kumar, R. Vijayvergiya, and S. Dogra, "Metabolic syndrome in psoriatic arthritis patients: a cross-sectional study," International Journal of Rheumatic Diseases, vol. 16, no. 6, pp. 667-673, 2013.

[33] N. Frey, T. Hugle, S. S. Jick, C. R. Meier, and J. Spoendlin, "Hyperlipidaemia and incident osteoarthritis of the hand: a population-based case-control study," Osteoarthritis and Cartilage, vol. 25, no. 7, pp. 1040-1045, 2017.

[34] S. Monira Hussain, Y. Wang, F. M. Cicuttini et al., "Incidence of total knee and hip replacement for osteoarthritis in relation to the metabolic syndrome and its components: a prospective cohort study," Seminars in Arthritis and Rheumatism, vol. 43, no. 4, pp. 429-436, 2014.

[35] G. Engstrom, M. Gerhardsson de Verdier, J. Rollof, P. M. Nilsson, and L. S. Lohmander, "C-reactive protein, metabolic syndrome and incidence of severe hip and knee osteoarthritis. A population-based cohort study," Osteoarthritis and Cartilage, vol. 17, no. 2, pp. 168-173, 2009.

[36] C. D. Han, I. H. Yang, W. S. Lee, Y. J. Park, and K. K. Park, "Correlation between metabolic syndrome and knee osteoarthritis: data from the Korean National Health and Nutrition Examination Survey (KNHANES)," BMC Public Health, vol. 13, no. 1, p. 603, 2013. 
[37] D. Xie, J. Wei, C. Zeng et al., "Association between metabolic syndrome and knee osteoarthritis: a cross-sectional study," BMC Musculoskeletal Disorders, vol. 18, no. 1, p. 533, 2017.

[38] M. Garcia-Gil, C. Reyes, R. Ramos et al., "Serum lipid levels and risk of hand osteoarthritis: the Chingford Prospective Cohort Study," Scientific Reports, vol. 7, no. 1, p. 3147, 2017.

[39] M. Zhou, Y. Guo, D. Wang et al., "The cross-sectional and longitudinal effect of hyperlipidemia on knee osteoarthritis: results from the Dongfeng-Tongji cohort in China," Scientific Reports, vol. 7, no. 1, article 9739, 2017.

[40] R. Inoue, Y. Ishibashi, E. Tsuda et al., "Medical problems and risk factors of metabolic syndrome among radiographic knee osteoarthritis patients in the Japanese general population," Journal of Orthopaedic Science, vol. 16, no. 6, pp. 704-709, 2011.

[41] T. Stürmer, Y. Sun, S. Sauerland et al., "Serum cholesterol and osteoarthritis. The baseline examination of the Ulm Osteoarthritis Study," The Journal of Rheumatology, vol. 25, no. 9, pp. 1827-1832, 1998.

[42] S. Onuora, "Metabolic syndrome and risk of knee OA," Nature Reviews Rheumatology, vol. 13, no. 5, p. 257, 2017.

[43] W. de Munter, M. H. van den Bosch, A. W. Slöetjes et al., "High LDL levels lead to increased synovial inflammation and accelerated ectopic bone formation during experimental osteoarthritis," Osteoarthritis and Cartilage, vol. 24, no. 5, pp. 844-855, 2016.

[44] R. G. Lahita, E. Rivkin, I. Cavanagh, and P. Romano, "Low levels of total cholesterol, high-density lipoprotein, and apolipoprotein $\mathrm{A} 1$ in association with anticardiolipin antibodies in patients with systemic lupus erythematosus," Arthritis and Rheumatism, vol. 36, no. 11, pp. 1566-1574, 1993.

[45] J. R. Bush, N. G. Berube, and F. Beier, "A new prescription for growth? Statins, cholesterol and cartilage homeostasis," Osteoarthritis and Cartilage, vol. 23, no. 4, pp. 503-506, 2015.

[46] A. Yamashita, M. Morioka, H. Kishi et al., "Statin treatment rescues FGFR3 skeletal dysplasia phenotypes," Nature, vol. 513, no. 7519, pp. 507-511, 2014. 